\title{
Influência das características pessoais do gerente de projetos no desempenho dos projetos
}

\author{
Influence of the project manager's personal characteristics on \\ project performance
}

\author{
Ralf Luis de Moura ${ }^{1}$ \\ Teresa Cristina Janes Carneiro ${ }^{1}$ \\ Bruna Demoner Diniz ${ }^{1}$
}

\begin{abstract}
Resumo: Projetos fazem parte das atividades organizacionais e têm impacto direto nos resultados e no desempenho das organizações. A melhoria no desempenho da Gestão dos Projetos pode trazer efeitos positivos para as organizações. O Gerente de Projetos, como responsável final pelos resultados dos projetos, tem em suas características pessoais fatores que podem impactar o Desempenho dos Projetos. Este estudo teve como objetivo, analisar as características pessoais do Gerente de Projetos em relação a seus efeitos no Desempenho dos Projetos, através de uma pesquisa empírica com 244 gerentes de projetos. Os resultados mostraram que Habilidades, Conhecimentos e Atitudes afetam diretamente o Desempenho dos Projetos e que os Traços de Personalidade não têm efeito direto, porém indireto, por afetarem as Atitudes. Os resultados ainda mostraram que a certificação em Gestão de Projetos não impacta diretamente o Desempenho dos Projetos, mas tem um efeito moderador nas relações entre Habilidades e Conhecimentos do Gerente de Projetos e o Desempenho da Gestão do Projeto.
\end{abstract}

Palavras-chave: Gerente de projetos; Competências; Personalidade; Desempenho.

\begin{abstract}
Projects form part of organizational activities and have a direct impact on the organization's results and performance. Improving project management performance can have a positive effect on an organization. Being ultimately responsible for project results, the project manager has factors within its personal characteristics that may affect project performance. The purpose of this study is to analyse the project manager's personal characteristics in relation to its effects on project performance using an empirical survey of 244 project managers. The results show that skills, knowledge and attitudes directly affect project performance and that personality traits do not have direct effects, but indirectly affect attitudes. The results also show that certification in project management does not directly affect project performance but has a moderating effect on the relationship between the project manager's skills and knowledge and project management performance.
\end{abstract}

Keywords: Project manager; Skills; Personality; Performance.

\section{Introdução}

Projetos são parte das atividades organizacionais e surgem nas organizações por vários motivos como: demandas de mercado, oportunidades ou necessidades estratégicas, avanços tecnológicos e requisitos legais, gerando impactos direto nos resultados da organização (Scott-Young \& Samson, 2008), motivo pelo qual muito ainda se pesquisa sobre projetos, sobre desempenho de projetos e sobre seus antecedentes (Anantatmula, 2015).

Segundo Kerzner (2010), grande parte do desempenho de um projeto pode ser atribuída às características pessoais do Gerente de Projetos (GP). Alguns teóricos destacam que aspectos comportamentais como Atitudes (Kerzner, 2010), Conhecimentos e Habilidades interpessoais (Lechler, 1998; Posner, 1987)

associados ao GP relacionam-se com o desempenho dos projetos (El-Sabaa, 2001; Thomas \& Mengel, 2008; Kerzner, 2010; Thal \& Bedingfiel, 2010). Porém não há um consenso ou uniformidade nos métodos de mensuração das características pessoais dos Gerentes de Projetos.

Segundo o PMI's Industry Growth Forecast, entre 2010 e 2020, 15,7 milhões de novos cargos de GP serão criados globalmente apenas nos sete principais ramos da indústria (PMI, 2012). Em 2015, no Brasil, o crescimento previsto de profissionais de Gestão de Projetos era de 2,2\%, mesmo em um cenário de recessão, segundo o Global Job Report (PMI, 2013). Esses números dão indícios da crescente importância do profissional de Gestão de Projetos nas organizações

\footnotetext{
${ }^{1}$ Programa de Pós-graduação em Administração - PPGADM, Universidade Federal do Espirito Santo - UFES, Av. Fernando Ferrari, 514, Goiabeiras, CEP29075-910, Vitória, ES, Brasil, e-mail: ralfmoura@gmail.com; teresa.janes.carneiro@gmail.com; bddiniz@gmail.com Recebido em Nov. 11, 2016 - Aceito em Maio 1, 2017
} 
(Hurt \& Thomas, 2009), mostrando a relevância e a importância do estudo das características desse profissional.

As organizações atribuem ao GP a responsabilidade final pela condução e entrega de resultados de seus projetos. Dentre as suas atribuições estão a responsabilidade de coordenar e integrar as atividades em várias linhas técnicas e funcionais além de gerenciar as comunicações entre as partes interessadas. Segundo o PMI (2012), o GP executa suas atividades diárias gerenciando o escopo do projeto, o tempo, riscos, custo, qualidade, relacionamentos com fornecedores, entre outros. Para tal, é necessário que o GP tenha habilidades técnicas, bem como a gestão de equipe, habilidade de negociação, perspicácia financeira e de negócios, combinadas com um entendimento da política de organização para atender os objetivos do projeto e para atender ou exceder as expectativas das partes interessadas (PMI, 2012). Kerzner (2010) afirma que profissionais de Gestão de Projetos precisam ter habilidades comportamentais e profissionais compatíveis com a função.

Personalidade pode ser definida como aspectos externos ou visíveis das características de um indivíduo. São atributos de um indivíduo que definem como esse indivíduo é visto pelos outros. O termo personalidade engloba uma série de qualidades sociais, subjetivas e emocionais (Schultz \& Schultz, 2009). Allport (1961) propõe a compreensão da personalidade humana por meio da análise de Traços de Personalidade que, combinados entre si, geram diferentes personalidades. Segundo McCrae \& John (1992), Traços de Personalidade são as dimensões básicas da personalidade ou as formas mais significativas e estáveis nas quais os indivíduos diferem entre si em seus estilos emocionais, interpessoais, experienciais, atitudinais e motivacionais.

O GP é o responsável pela condução de todos os aspectos que envolvem o projeto e, como tal, tem poder de interferência no desempenho e nos seus resultados. Dessa forma, entende-se que há a necessidade de entender os fatores que afetam o seu desempenho como gestor. Com base nessas constatações, surgem os seguintes questionamentos: Quanto do resultado final de um projeto pode ser imputado às características pessoais do responsável pela gestão do projeto? Que características pessoais associadas às competências profissionais do GP têm mais peso nessa relação?

O objetivo deste estudo foi analisar as características pessoais do Gerente de Projetos em relação a seus efeitos no Desempenho dos Projetos, explorando essas relações. Para buscar respostas, foram levantadas na literatura as principais Habilidades, Conhecimentos e Atitudes (CHA) associadas ao GP e, com base em tais características pessoais, um modelo estrutural foi criado, relacionando-as ao Desempenho de Projetos.

Este trabalho está organizado da seguinte forma: A seção seguinte apresenta o modelo de pesquisa e a conceituação teórica, seguida dos procedimentos metodológicos e pela apresentação dos resultados. A análise dos resultados é então desenvolvida e discutida e, por fim, as considerações finais são apresentadas, abrindo novas oportunidades de estudo e discussão acerca das implicações práticas dos resultados.

\section{Fundamentação teórica}

\subsection{Desempenho de projetos}

Muitos projetos ainda falham em atender as expectativas (Williams, 2005), e por isso muito se discute sobre o sucesso de projetos. Todavia, há um consenso de que a determinação do sucesso de um projeto é uma tarefa complexa (Milis \& Mercken, 2002). Os critérios de sucesso vêm evoluindo (Kerzner, 2010) desde a década de 1960, quando eram considerados apenas aspectos técnicos, passando pelo triângulo de ferro, na década de 1970 (prazo-custo-escopo-qualidade), satisfação do cliente, na década de 1980, impactos organizacionais, na década de 1990 (O’Brochta, 2002; Ika, 2009), até os critérios mais recentes, que consideram os impactos sociais e ambientais (Kerzner, 2010).

Trabalhos como os Wit (1988), Atkinson (1999), Lim \& Mohamed (1999), Cooke-Davies (2002), Kerzner (2010), Anantatmula (2015) discutiram critérios de sucesso de projetos sob variadas perspectivas. Nesses trabalhos estão incluídos os aspectos técnicos considerados de medição simples e aspectos subjetivos muitas vezes intangíveis e difíceis de mensurar (Freeman \& Beale, 1992). Wit (1988) e Cooke-Davies (2002) distinguem os critérios de sucesso em duas grandes categorias: critérios de sucesso dos Projetos e critérios de sucesso na Gestão dos Projetos. Freeman \& Beale (1992) defendem que os critérios com maior carga de subjetividade, tais como impactos organizacionais, percepção de satisfação dos stakeholders, entre outros, estão relacionados ao sucesso do projeto, enquanto que os aspectos técnicos relacionados à tripla restrição (prazo, escopo e qualidade) seriam critérios de medição relacionados ao sucesso da Gestão do Projeto.

Portanto, para medir o sucesso da Gestão de Projetos são utilizados critérios técnicos tangíveis como variáveis de medição. Os aspectos técnicos em geral incluem as quatro dimensões presentes na versão clássica do triângulo de ferro: Prazo, Custo, Escopo e Qualidade (PMI, 2012; Ika, 2009). Esses critérios técnicos são a base das pesquisas relacionadas ao sucesso nos projetos (Atkinson, 1999) apresentadas neste trabalho e medem não o sucesso dos projetos mas o sucesso da Gestão dos Projetos. Por ser uma proxy do desempenho, neste trabalho o sucesso na Gestão de Projetos e Desempenho dos Projetos são termos intercambiáveis.

Segundo Isik et al. (2009), o gerenciamento do cronograma habilita o projeto a ser completado dentro do prazo estabelecido, o gerenciamento do custo inclui, entre outras atividades, o controle do 
custo, que permite o projeto ser concluído o mais próximo possível do valor inicialmente orçado. O controle da qualidade está diretamente relacionado ao cumprimento dos requisitos e à satisfação do cliente. O PMI (2012) afirma que o gerenciamento do escopo inclui atividades que visam garantir que o projeto entregue todos os requisitos acordados.

\subsection{Características pessoais}

Características pessoais são objeto de vários estudos no meio acadêmico. Pérez \& Rodríguez del Bosque (2013) estudaram as características pessoais de consumidores em relação à percepção da responsabilidade social corporativa, Grygus \& Prusik (2015) analisaram as características pessoais de profissionais de saúde, Bordovskaia \& Kostromina (2013) pesquisaram as características pessoais de estudantes. Cada um dos estudos citados buscou encontrar características comuns, de forma a entender o comportamento desses indivíduos.

As características pessoais de um profissional aparecem na literatura científica como competências. Zarifian (2003) entende competência como sendo a capacidade de tomar iniciativa; a capacidade de ter o entendimento prático de situações, baseado no conhecimento, e a capacidade de mobilizar uma rede de atores e fazer com que compartilhem ações e responsabilidades. Le Boterf (2003) defende que competência é resultado de um conjunto de recursos e características pessoais tais como: conhecimentos, habilidades, qualidades, experiências, capacidades cognitivas, entre outros. Dutra (2001), Fleury (2002), Lustri et al. (2005) e Durand (2006) descrevem competência em três dimensões: conjunto de Conhecimentos, Habilidades e Atitudes, que englobam questões técnicas, cognição e comportamento.

Este estudo está baseado no entendimento de competência como composta das seguintes dimensões: Conhecimentos, Habilidades e Atitudes (CHA), as quais indicam o que o profissional sabe, sua vontade de querer fazer ou de fazer acontecer e sua capacidade de saber como fazer (Ajzen, 2005).

Conhecimentos são conjuntos estruturados de informação assimilada que permitem o entendimento do mundo, englobando acesso aos dados e a habilidade de transformá-los em informação utilizável (Pires, 2005). Habilidades são a capacidade de agir de forma concreta de acordo com os objetivos predefinidos, relacionadas com empirismo. Elas são relacionadas ao saber fazer algo ou à capacidade de fazer uso produtivo do conhecimento (Pires, 2005). Atitudes referem-se à tendência psicológica, à memória e à avaliação de objetos ou entidades (Bagozzi et al., 2002). Ajzen (2005) afirma que Atitudes são características hipotéticas latentes que só podem ser inferidas a partir de sugestões externas observáveis. Atitude trata essencialmente da motivação (vontade, comprometimento, determinação) do indivíduo, da sua proatividade na mobilização dos recursos de que dispõe para aplicação na situação que enfrenta (Durand, 2006).

\subsection{Traços de personalidade}

Personalidade pode ser definida como sendo a organização dinâmica dentro dos sistemas psicofísicos do indivíduo que determina seu comportamento e pensamento (Allport, 1961). Personalidade é uma área de estudos muito ampla que tem sido conceituada por meio de diversas perspectivas teóricas que contribuem para o entendimento das diferenças individuais relacionadas ao comportamento e às experiências dos indivíduos (John \& Srivastava, 1999).

Nicholson (2000) define personalidade como “permanência do caráter". Já Buchaman \& Huczynski (1997) definem personalidade como "as qualidades psicológicas que influenciam os padrões comportamentais, típicos de um indivíduo, de uma forma distintiva e consciente, através de diferentes situações ao longo do tempo". Schultz \& Schultz (2009) defendem que personalidade são os aspectos externos ou visíveis das características de um indivíduo.

Personalidade pode ser medida por meio de múltiplos instrumentos. Um instrumento sucinto de medição que permite que os pesquisadores entendam de forma simplificada, porém abrangente, determinados domínios das características de personalidade (John \& Srivastava, 1999; Gosling et al., 2003) é conhecido como Big-Five Traits.

Esse instrumento contempla cinco domínios, conhecidos como Traços de Personalidade, que representam a personalidade em um amplo nível de abstração, sendo cada dimensão um sumário de um conjunto de características específicas de personalidade (John \& Srivastava, 1999). O Big-Five é uma taxonomia utilizada no ramo da Psicologia (Goldberg, 1990) que, embora não seja aceita universalmente, aparece constantemente nos principais estudos relacionados à personalidade (Gosling et al., 2003). O modelo Big-Five organiza as características de um indivíduo em cinco domínios: abertura às experiências, conscienciosidade, amabilidade, neuroceticismo e extroversão (Soto \& John, 2009).

Extroversão demonstra o nível de conforto com relacionamentos. Os extrovertidos tendem a ser gregários, assertivos e sociáveis. A amabilidade refere-se à propensão de um indivíduo para amar outros. Pessoas altamente amáveis são cooperativas e confiantes. A conscienciosidade é uma medida de confiabilidade. Uma pessoa altamente consciente é responsável, organizada, confiável e persistente. O neuroceticismo (instabilidade emocional) mostra a incapacidade de suportar o stress de uma pessoa. Aqueles com alta instabilidade emocional tendem a ficar nervosos, ansiosos, deprimidos e inseguros. A abertura às experiências considera uma gama de interesses e a fascinação com a novidade. Pessoas abertas são criativas, curiosas e artisticamente sensíveis (Gosling et al., 2003; Soto \& John, 2009). 


\subsection{Características pessoais do GP}

O GP é o profissional diretamente responsável pelos resultados dos projetos, o que denota sua importância (PMI, 2012). Com base em tal constatação, cresce nas empresas o interesse pelo mapeamento das características desse profissional (Crawford, 2005). Dentre as características estão as competências profissionais, experiência profissional e a personalidade do GP.

Características pessoais se aplicam a qualquer indivíduo ou profissional. Para o GP foi necessário buscar estudos específicos para dar sustentação teórica à pesquisa. Características pessoais do GP é um tema largamente explorado no meio científico. Gaddis (1959) em meados do século passado já discutia o que um GP na indústria de tecnologia fazia, que tipo de profissional deveria ser e quais treinamentos eram pré-requisitos para seu sucesso.

Sbragia et al. (1986), em seu estudo sobre o GP, seu papel e habilidades, afirmam que boa parte do sucesso do projeto depende da posse de um conjunto peculiar de aptidões por parte do GP e as classificam em três famílias: conhecimentos (o que o indivíduo conhece), as atitudes (o que ele pensa a respeito de si, de seu trabalho e de outros aspectos do seu ambiente) e as habilidades (o que ele sabe fazer).

Diferentes estudos analisam a relação entre o perfil do GP e o sucesso dos projetos (Haggerty, 2000; Lampel, 2001; Brill et al., 2006; Fisher, 2011), outros comparam gerentes funcionais e gerentes de projetos em termos de perfil, atributos e habilidades (El-Sabaa, 2001) e ainda identificam as áreas de conhecimento e o perfil requerido para o GP em áreas como a construção civil (Fotwe \& McCaffer, 2000; Lampel 2001). Os estudos sobre esse tema trazem diferentes formas de mensuração, envolvendo diferentes indicadores, e parte do trabalho foi identificar e consolidar as principais características discutidas no meio científico. Para tal, foi realizado um levantamento bibliográfico cujo resultado apresenta-se consolidado e sintetizado como um questionário descritos em quadros no Apêndice A. As características pessoais identificadas são a base para a formulação dos constructos: Conhecimentos, Habilidades, Atitudes e Traços de Personalidade.

O modelo proposto se baseia nos pressupostos defendidos por teóricos de que as características compostas por: Habilidades, Conhecimentos e Atitudes do GP têm impacto direto no Desempenho dos Projetos (Haggerty, 2000; Lampel, 2001; Brill et al., 2006; Fisher, 2011; Fotwe \& McCaffer, 2000; El-Sabaa, 2001; Hurtz \& Donovan, 2000). Baseado nos argumentos teóricos apresentados, foram definidas as hipóteses:

\section{- H1a: As Atitudes do GP afetam o Desempenho} do Projeto;

- H1b: As Habilidades do GP afetam o Desempenho do Projeto;

\section{- H1c: Os Conhecimentos do GP afetam o Desempenho do Projeto.}

Traços de Personalidade aparecem em estudos científicos ligados ao desempenho do trabalho (Hurtz \& Donovan, 2000; Barrick \& Mount, 1991; Barrick et al., 1993) e são considerados características relevantes no estudo do desempenho profissional. O desempenho profissional do GP pode afetar os resultados do seu trabalho, compondo a seguinte hipótese:

\section{- H2a: Os Traços de Personalidade do GP afetam o Desempenho do Projeto.}

A literatura especializada relaciona Traços de Personalidade e Atitudes. Os Traços de Personalidade, no contexto das características pessoais (Durand, 2006), estão ligados à tendência psicológica, à memória $\mathrm{e}$ à avaliação de objetos ou entidades (Bagozzi et al., 2002), vinculadas a Atitudes e Comportamentos. Baseado nessa abordagem teórica, espera-se que os Traços de Personalidade afetem as Atitudes do GP formando a hipótese:

\section{- H2b: Os Traços de Personalidade do GP afetam as atitudes do GP.}

\subsection{Certificações}

Turner \& Huemann (2001) afirmam que a competência na área de Gestão de Projetos é baseada em conhecimento e experiência. Segundo os autores, a oferta de programas de educação de gerenciamento de projetos formais é essencial para o desenvolvimento das competências desejadas. Para determinar se os padrões de conhecimento e habilidades desejados foram atingidos por um profissional, é necessário realizar uma avaliação da qualificação desse profissional (Hartman \& Skulmoski, 1999). Portanto, a finalidade de uma certificação é reconhecer as habilidades e conhecimentos de um profissional.

O propósito das certificações em Gestão de Projetos é prover o reconhecimento da capacidade profissional do GP (Hartman \& Skulmoski, 1999; Turner \& Huemann, 2001), comprovando seus conhecimentos, experiência e habilidades em Gestão de Projetos. Existem várias certificações no mercado, algumas voltadas para testes de conhecimento, oferecidas por associações de profissionais como, por exemplo, o PMP - Project Management Professional do PMI - Project Management Institute; a certificação oferecida pelo Australian Institute of Project Management - AIPM, que é baseada nos padrões nacionais de competência australianos (AIPM, 1996); a certificação da Association for Project Management - APM, no Reino Unido, que usa seu próprio conjunto de conhecimento; Prince2 - Projects in Controlled Environments, oferecido pela APMG International; até certificações que atestam, além dos 
conhecimentos, as habilidades e experiências do GP, como a oferecida pela International Project Management Association - IPMA, baseada no documento ICB (IPMA Competence Baseline) (Artto, 2000).

Profissionais certificados nessas metodologias supostamente tenderiam a conhecer melhor suas metodologias e, por consequência, a ter melhores resultados na gestão de seus projetos (PMI, 2012). Segundo essas metodologias, a certificação afeta os Conhecimentos e Habilidades do GP e, nesse caso, supostamente, teriam um efeito moderador no Desempenho da Gestão do Projeto (Hartman \& Skulmoski, 1999). Baseado nessas afirmações foram definidas as hipóteses:

- H3a: A certificação em Gestão de Projetos tem um efeito moderador na relação entre o Conhecimento do GP e o Desempenho do Projeto;

- H3b: A certificação em Gestão de Projetos tem um efeito moderador na relação entre as Habilidades do GP e o Desempenho do Projeto.

\section{Metodologia}

As hipóteses e bases teóricas geraram o modelo hipotético apresentado na Figura 1. Pesquisas que envolvem características pessoais normalmente formam um constructo Competência, a partir de Conhecimentos, Habilidades e Atitudes (Brandão \& Borges-Andrade, 2007; Brandão, 2012; Carbone et al., 2009).

Este estudo optou por manter as características pessoais do GP como relacionando-se diretamente ao desempenho do projeto para manter as relações mais explícitas e para que se pudesse testar o impacto e os efeitos de moderação diretamente, sem um constructo de segunda ordem Competência. Ademais, como os constructos Habilidades e Conhecimentos são formativos e Atitudes é reflexivo, não seria

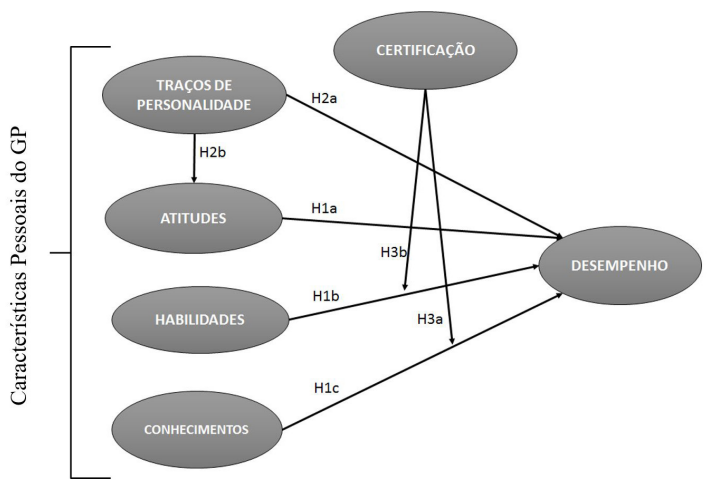

Figura 1. Modelo estrutural hipotético. Fonte: Autores (2016). possível montar o modelo dessa forma (Hair et al., 2014, p. 231).

\subsection{Operacionalização das variáveis e coleta de dados}

Atitudes, Habilidades, Conhecimentos e Traços de Personalidades são constructos que teoricamente impactam o Desempenho dos Projetos. As 31 variáveis levantadas na literatura (Apêndice A) dividem-se por esses constructos, sendo 8 variáveis para Conhecimentos, 8 variáveis para Habilidades, 5 variáveis para Atitudes, 5 indicadores para os Traços de Personalidade, 4 variáveis para o constructo de Desempenho e uma variável dicotômica para certificação.

Desempenho foi medido pelo sucesso dos projetos (Q4n), de forma reflexiva. O sucesso de projetos foi decomposto em quatro tipos de sucesso: custo, prazo, qualidade e escopo. O modelo de mensuração de Atitudes, formado pelas variáveis Q3n, é reflexivo, atitudes são normalmente vistas como predisposição a responder de uma forma favorável ou desfavorável a um objeto e são tipicamente medidas através de indicadores reflexivos (Jarvis et al., 2003). Os modelos de mensuração formados pelas variáveis Q1n, Q2n, Q5n são formativos e formam os constructos Conhecimentos, Habilidades e Traços de Personalidades. As questões inseridas nos questionários foram formuladas de forma a atender os requisitos reflexivos e formativos.

A coleta de dados foi realizada por meio de um questionário online dividido em quatro partes. As três partes iniciais com perguntas sobre Conhecimentos, Habilidades, Atitudes e Traços de Personalidade do GP, respectivamente. A quarta e última parte do questionário composta por questões acerca do sucesso dos três últimos projetos sob as lentes custo, qualidade, escopo e prazo.

Cada pergunta do questionário foi vinculada a uma das 26 variáveis identificadas no referencial teórico, utilizando escala do tipo Likert de 5 pontos. As quatro perguntas relativas a sucesso utilizaram uma escala numérica de 0 a 3, que representa a quantidade de projetos concluídos com sucesso considerando os últimos 3 projetos dentro das perspectivas custos, escopo, qualidade e prazos. No início do questionário foi realizada uma pergunta acerca da certificação em Gestão de Projetos.

A pesquisa foi realizada com profissionais que atuam com Gestão de Projetos no Brasil, durante os meses de setembro e dezembro de 2015. O questionário foi enviado para associações de gerenciamento de projetos dos estados da região sudeste do Brasil. Foram coletadas 244 respostas de Gerentes de Projetos com idade entre 25 e 60 anos, $96 \%$ com curso superior completo, distribuídos nas áreas de tecnologia da informação (61\%), engenharia (23\%), administração $(11 \%)$ e outras $(5 \%)$. Do total de profissionais que responderam à pesquisa, $79 \%$ gerenciam projetos há mais de 2 anos, sendo que $60 \%$ do total, há mais de 5 anos, $66 \%$ do total trabalham no setor privado 
e 34\% no setor público. O percentual de Gerentes de Projetos certificados em Gestão de Projetos é de $40 \%, 75 \%$ desses são do setor privado.

\subsection{Modelos de mensuração}

A amostra utilizada possui 244 observações, sendo, portanto, suficientes para atender os requisitos mínimos de tamanho da amostra, que deve ser 10 vezes maior que o maior número de indicadores dos constructos formativos (Hair et al., 2014, p. 20). Os constructos Conhecimentos e Habilidades possuem 8 indicadores, o que indica uma necessidade mínima de 80 observações. Para a análise de moderação, a quantidade de profissionais certificados e não certificados também ultrapassa as 80 observações.

O modelo hipotético proposto foi investigado a partir da técnica de Modelagem de Equações Estruturais (PLS-SEM - Partial Least Squares Structural Equation Modeling), utilizando-se o software SmartPLS3 (Ringle et al., 2015). PLS-SEM foi utilizado para avaliação do modelo de mensuração reflexivo dos constructos Desempenho e Atitudes e para a avaliação dos constructos formativos Conhecimentos, Habilidades e Personalidade.

No Constructo reflexivo Desempenho, a confiabilidade da consistência interna apresentou a confiabilidade composta de 0,8911, e o constructo Atitudes, 0,8531, segundo Nunnally \& Bernstein (1994), dentro da faixa satisfatória, entre 0,700 e 0,900 . A validade convergente, que é o indicador de confiabilidade, foi medida a partir das cargas fatoriais dos indicadores reflexivos. A carga fatorial do indicador Q3a apresentou 0,6302 , ou seja, abaixo de 0,708 , como indicado na literatura (Hair et al., 2014, p. 103), porém, ao se remover esse indicador, o constructo perderia validade de conteúdo e sua remoção não apresentou grande aumento na média da variância extraída (AVE) $(0,5389$ para 0,6075$)$ ou na confiabilidade composta $(0,8531$ para 0,8608$)$, dessa forma, optou-se por manter esse indicador (Hair et al., 2014, p. 102). A validade convergente ainda é avaliada pelo índice AVE, que para os constructos Desempenho e Atitudes (0,5647 e 0,6719) comprimiu os critérios de Validade Convergente, a qual, segundo Hair et al. (2014, p. 103), deve se apresentar acima de 0,50.

A validade discriminante dos constructos reflexivos é avaliada a partir da análise das cargas cruzadas e do critério de Fornell-Larcker, no qual a raiz quadrada de AVE deve ser maior que as correlações com os demais constructos (Hair et al., 2014, p. 105). Estudos recentes examinaram os critérios de cargas cruzadas e Fornell-Larcker e indicaram que em determinadas situações eles podem não ser confiáveis (Henseler et al., 2015). Para contornar esse problema, uma nova técnica conhecida como HTMT (Heterotrait-Monotrait ratio) foi proposta e está disponível no SmartPLS3. Para o critério HTMT foi utilizado o procedimento bootstrapping, com 5 mil interações, no qual se derivou o intervalo de confiança, neste trabalho, de 95\%. Após a execução, nenhum constructo indicou problemas de validade discriminante. Por ser uma técnica nova, as validações tradicionais ainda são consideradas padrões para análise de validade discriminante e foram também analisadas (Hair et al., 2015, p. 119), sendo os critérios de validade discriminante considerados atendidos. Com base nesses resultados, todos os indicadores reflexivos do constructo Desempenho e Atitudes foram mantidos.

Para as avaliações dos modelos de mensuração formativos foi realizada a avaliação da multicolinearidade realizada a partir do Fator de Inflação da Variância (VIF) que, segundo Hair et al. (2011, p. 145), deve ser inferior a 5,0. Todos indicadores apresentaram valores de VIF inferiores a 5,0 variando de 1,211, do indicador Q5b, a 2,347, do indicador Q4c.

A relevância e significância dos pesos foram avaliadas a partir da função bootstrapping com 5 mil interações. Somente os indicadores Q1a, Q1c, Q5a, Q5b e Q5c aprestaram pesos significantes, os indicadores $\mathrm{Q} 1 \mathrm{~b}$, Q1d, Q1e, Q1f, Q1g, Q1h, Q2a, Q2c, Q2d, Q2e, Q2f, Q2h apresentaram carga fatorial acima de 0,500 e os indicadores Q2b, Q2g, Q5d apresentaram carga fatorial abaixo de 0,500 , porém significante a 0,01 . Todos pesos de todos os indicadores formativos apresentaram importância absoluta e/ou relativa (Hair et al., 2014, p. 127-129) e, por esse motivo, todos foram mantidos no modelo.

\section{Análise do modelo estrutural e resultados}

Após o ajuste dos modelos de mensuração, o modelo estrutural foi analisado através dos coeficientes de caminho apresentados na Tabela 1 . Apenas o coeficiente de caminho de Personalidade para Desempenho não foi significante. Porém, ao se analisar os efeitos totais percebe-se que ao afetar as Atitudes, a Personalidade tem um efeito indireto no Desempenho. O efeito apresentado foi de 0,1608 , com um P-valor de 0,0378 , ou seja, significativo.

A Figura 2 ilustra os coeficientes de caminho após a análise do modelo proposto.

$\mathrm{O}$ coeficiente de determinação $\mathrm{R}^{2}$ do constructo Atitudes foi de 0,736 , os Traços de Personalidade são capazes de explicar $73,6 \%$ das Atitudes do GP, e o do constructo Desempenho foi de 0,399. As competências são capazes de explicar cerca de 39,99\% do Desempenho do Projeto. As competências representadas por Habilidades, Conhecimentos e Atitudes apresentaram coeficientes de caminhos significativos e esses resultados permitem avaliar as hipóteses propostas. O Quadro 1 apresenta os resultados finais dos testes de hipótese analisados neste estudo. 


\subsection{Teste de hipóteses para diferença de médias}

Para confirmar se os coeficientes de caminhos não são iguais e dessa forma permitir a comparação entre eles, foi realizado um teste de hipótese da diferença das médias entre os erros padrões gerados na execução da técnica bootstrapping. Os resultados dos testes são apresentados na Tabela 2, na qual observa-se que as hipóteses de igualdade das médias foram rejeitadas. Portanto, é possível a realização da comparação das médias.

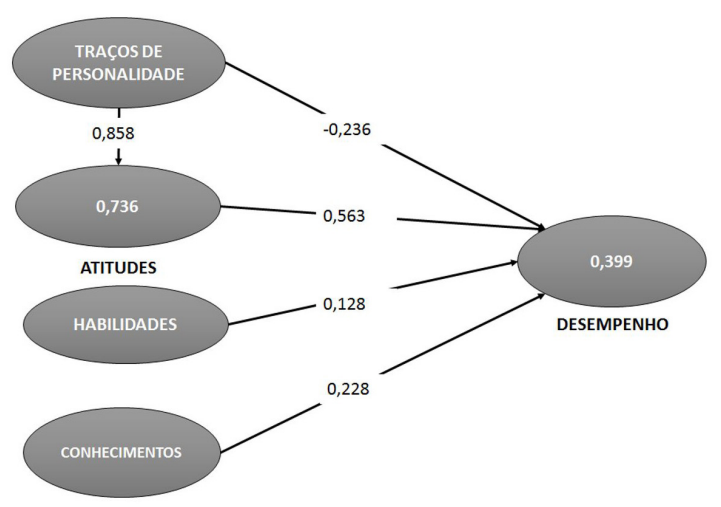

Figura 2. Coeficientes de caminho. Fonte: Autores (2016).

\subsection{Análise da moderação}

Apesar de não ter sido inicialmente considerada uma hipótese desta pesquisa, a primeira análise realizada foi a de influência da variável dicotômica Q6a (Certificação) no Desempenho da Gestão de Projetos; ela foi adicionada como variável preditora com o objetivo de averiguar se haveria algum impacto direto no Desempenho do Projeto e para, a partir do resultado, permitir inferências sobre os Conhecimentos e as Habilidades do GP. Ao se acrescentar essa variável, uma pequena mudança ocorreu no valor de $\mathrm{R}^{2}$ do constructo, que foi de 0,399 para 0,398. E os coeficientes de caminho mudaram, conforme apresentado na Tabela 3.

O coeficiente de caminho da variável certificação não foi significativo, porém percebe-se que os demais coeficientes foram ligeiramente influenciados pela entrada dessa variável.

A análise de moderação em relação ao Conhecimento foi o segundo teste realizado e o valor de $\mathrm{R}^{2}$ foi para 0,414 . Os demais resultados estão apresentados na Tabela 4 e na Figura 3.

$\mathrm{O}$ efeito moderador se mostrou significativo entre Conhecimento e Desempenho. E com influência no coeficiente de determinação.

A terceira análise de moderação foi realizada entre Habilidades e Desempenho da Gestão do Projeto. $\mathrm{O}$ valor de $\mathrm{R}^{2}$ foi de 0,4003 . Os demais resultados estão apresentados na Tabela 5 e na Figura 4.

Quadro 1. Avaliação das hipóteses.

\begin{tabular}{|l|l|c|}
\hline & \multicolumn{1}{|c|}{ Hipótese } & Resultado \\
\hline $\mathrm{H}_{\mathrm{a}}$ & H1a: As Atitudes do GP afetam o Desempenho do Projeto & Suportada \\
\hline $\mathrm{H} 1_{\mathrm{b}}$ & H1b: As Habilidades do GP afetam o Desempenho do Projeto & Suportada \\
\hline $\mathrm{H} 1_{\mathrm{c}}$ & H1c: Os Conhecimentos do GP afetam o Desempenho do Projeto & Suportada \\
\hline $\mathrm{H} 2_{\mathrm{a}}$ & H2a: Os Traços de Personalidade do GP afetam o Desempenho do Projeto & Rejeitada \\
\hline $\mathrm{H} 2_{\mathrm{b}}$ & H2b: Os Traços de Personalidade do GP afetam as Atitudes do GP & Suportada \\
\hline
\end{tabular}

Fonte: Autores (2016).

Tabela 1. Coeficientes de caminho.

\begin{tabular}{lcc}
\hline \multicolumn{1}{c}{ Caminho } & Coeficiente de caminho & P-valor \\
\hline Atitudes - Desempenho & 0,563 & 0,0153 \\
Conhecimentos - Desempenho & 0,228 & 0,0006 \\
Habilidades - Desempenho & 0,128 & 0,0332 \\
Traços de Personalidade - Atitudes & 0,858 & 0,0000 \\
Traços de Personalidade - Desempenho & $-0,236$ & $\mathbf{0 , 9 7 8 7}$ \\
\hline
\end{tabular}

Fonte: Autores (2016).

Tabela 2. Teste t - Hipótese de Igualdade das Médias.

\begin{tabular}{lcccc}
\hline & Média & Desvio padrão & Erro & P-valor \\
\hline Atitudes / Conhecimentos & $-0,087$ & 0,131 & 0,006 & 0,000 \\
Conhecimentos / Habilidades & 0,071 & 0,131 & 0,006 & 0,000 \\
Habilidades / Atitudes & $-0,017$ & 0,128 & 0,006 & 0,004 \\
\hline
\end{tabular}

Fonte: Autores (2016). 
O efeito moderador se mostrou significativo entre Habilidades e Desempenho apenas a 0,10, com uma influência menor no coeficiente de determinação do Desempenho. O Quadro 2 consolida a avaliação das hipóteses de moderação.

\section{Discussões}

As características pessoais do GP explicam parcialmente o Desempenho dos Projetos, conforme demonstrado pelo coeficiente de determinação $\mathrm{R}^{2}=0,399$. Esse resultado era esperado, pois existem potencialmente outros elementos que podem influenciar o Desempenho dos Projetos. Na literatura podem ser encontrados fatores como, por exemplo: Metodologia de Gestão de Projetos, disponibilidade de recursos, influências externas, como questões econômicas, de governo e outros eventos fora do controle do GP, entre outros (Belassi \& Tukel, 1996; Cooke-Davies, 2002).

As hipóteses H1a, H1b, H1c não foram possíveis de serem rejeitadas, portanto há indícios empíricos de que as Atitudes, Habilidades e Conhecimentos do GP impactam no Desempenho do Projeto. Esses resultados estão alinhados com os vários estudos científicos que defendem que as competências e características pessoais do GP influenciam a Gestão do Projetos e em seus resultados (Haggerty, 2000; Lampel, 2001; Brill et al., 2006; Fisher, 2011; Fotwe \& McCaffer, 2000; El-Sabaa, 2001).

As Atitudes demonstram ter um peso superior $(0,563)$ no impacto sobre o Desempenho do Projeto, seguida dos Conhecimentos $(0,228)$ e Habilidades $(0,128)$. Os resultados indicam que melhoras nas

Tabela 3. Coeficientes de caminho após adição da variável certificação.

\begin{tabular}{lcc}
\hline \multicolumn{1}{c}{ Caminho } & Coeficiente de caminho & P-valor \\
\hline Atitudes -> Desempenho & 0,598 & 0,0203 \\
Conhecimentos -> Desempenho & 0,227 & 0,0002 \\
Habilidades -> Desempenho & 0,137 & 0,0137 \\
Traços de Personalidade -> Atitudes & 0,778 & 0,0000 \\
Certificação -> Desempenho & 0,050 & 0,5125 \\
\hline
\end{tabular}

Fonte: Autores (2016).

Tabela 4. Coeficientes de caminho Moderação - Conhecimento.

\begin{tabular}{lcc}
\hline \multicolumn{1}{c}{ Caminho } & Coeficiente de caminho & P-valor \\
\hline Atitudes -> Desempenho & 0,5136 & 0,0500 \\
Conhecimentos -> Desempenho & 0,2153 & 0,0005 \\
Habilidades -> Desempenho & 0,1485 & 0,0132 \\
Traços de Personalidade -> Atitudes & 0,8782 & 0,0000 \\
Certificação -> Desempenho & 0,0309 & 0,7688 \\
Efeito moderador & 0,1542 & 0,0045 \\
\hline
\end{tabular}

Fonte: Autores (2016).

Tabela 5. Coeficientes de caminho Moderação - Habilidades.

\begin{tabular}{lcc}
\hline \multicolumn{1}{c}{ Caminho } & Coeficiente de caminho & P-valor \\
\hline Atitudes -> Desempenho & 0,5428 & 0,0500 \\
Conhecimentos -> Desempenho & 0,2329 & 0,0002 \\
Habilidades -> Desempenho & 0,1328 & 0,0369 \\
Traços de Personalidade -> Atitudes & 0,8782 & 0,0000 \\
Certificação -> Desempenho & 0,379 & 0,7595 \\
Efeito moderador & 0,0680 & 0,0723 \\
\hline
\end{tabular}

Fonte: Autores (2016).

Quadro 2. Avaliação das hipóteses de moderação.

\begin{tabular}{|l|l|c|}
\hline & \multicolumn{1}{|c|}{ Hipótese } & Resultado \\
\hline H3 a & $\begin{array}{l}\text { H3a: A certificação em Gestão de Projetos tem um efeito moderador na relação entre } \\
\text { o Conhecimento do GP e o Desempenho da Gestão do Projeto }\end{array}$ & Suportada \\
\hline H3 & $\begin{array}{l}\text { H3b: A certificação em Gestão de Projetos tem um efeito moderador na relação entre } \\
\text { as Habilidades do GP e o Desempenho da Gestão do Projeto }\end{array}$ & Suportada \\
\hline
\end{tabular}

Fonte: Autores (2016). 


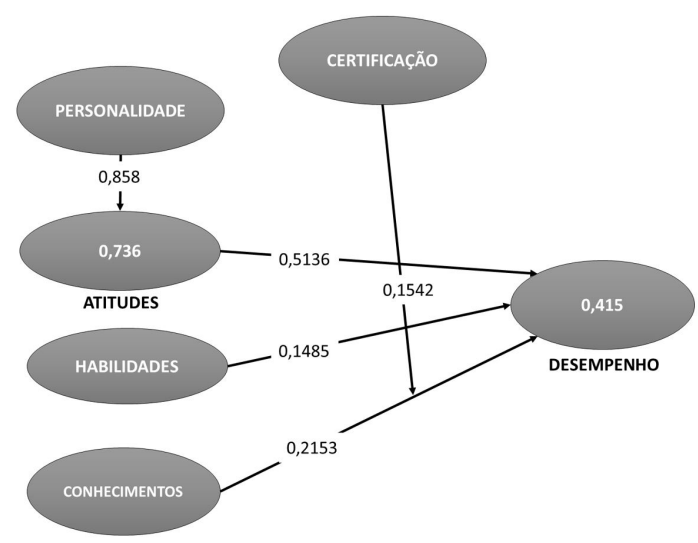

Figura 3. Coeficientes de caminho com moderação. Fonte: Autores (2016).

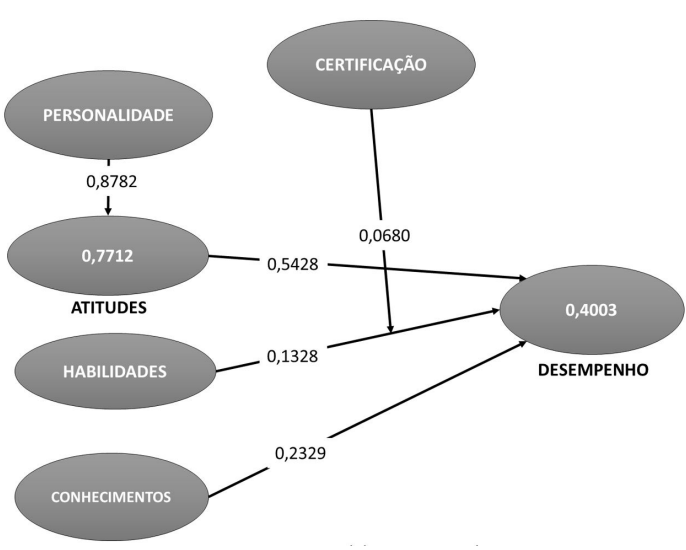

Figura 4. Coeficientes de caminho. Fonte: Autores (2016).

Atitudes e Conhecimentos geram maiores impactos no Desempenho dos Projetos. Habilidades que, representam a experiência profissional do GP (Quadro 1), têm menor efeito no Desempenho dos Projetos.

A hipótese H2a foi rejeitada, com isso é possível inferir que os Traços de Personalidade do GP não afetam o Desempenho do Projeto. Porém a hipótese $\mathrm{H} 2 \mathrm{~b}$ foi suportada, o que indica alinhamento com as bases teóricas sobre Atitudes (Durand, 2006; Bagozzi et al., 2002). Como os Traços de Personalidade do GP afetam as suas Atitudes, apresentam um efeito indireto no Desempenho do Projeto, e são, portanto, um fator importante a ser considerado.

Não é possível afirmar empiricamente que a certificação em Gestão de Projetos afeta diretamente o Desempenho dos Projetos. Porém o efeito moderador representado pelas hipóteses H3a e H3b não foi possível de ser rejeitado, logo a certificação em Gestão de Projetos tem um efeito moderador sobre os Conhecimentos e as Habilidades do GP. Esses resultados corroboram os estudos científicos que indicam que o uso e o Conhecimento de metodologias influenciam nas Habilidades e Conhecimentos dos Gerentes de Projetos e, consequentemente, nos resultados e Desempenho dos Projetos (Hartman \& Skulmoski, 1999).

A partir das conclusões realizadas neste estudo científico é possível afirmar que as Características Pessoais do GP têm efeitos relevantes sobre o Desempenho e, consequente, no resultado dos projetos. Processos de seleção e de capacitação desse profissional devem se concentrar em detectar e desenvolver essas características de forma a aumentar os resultados práticos de seus projetos e das estratégias organizacionais. Certificações em Gestão de Projetos não garantem diretamente melhorias no Desempenho dos Projetos, mas, por apresentarem um efeito moderador, elas podem servir como fatores impulsionadores de melhorias nas relações entre as Habilidades e os Conhecimentos do GP e, dessa forma, ter impactos nos seus resultados.

\section{Considerações finais}

Este estudo atingiu seu objetivo ao apresentar as competências do GP que influenciam o Desempenho dos Projetos. Os resultados mostraram indícios de que todos as características pessoais: Conhecimentos, Atitudes e Habilidades do GP têm impacto significativo e podem explicar parcialmente o Desempenho dos Projetos.

Das características pessoais, Atitudes, Conhecimentos e Habilidades têm efeitos diferentes no Desempenho dos Projetos e, dessa forma, conclui-se que devem ser priorizadas de forma diferente ao se buscar por melhorias no Desempenho dos Projetos. Atitudes devem ter prioridade, seguidas pelos Conhecimentos e, posteriormente, pelas Habilidades.

Dois pontos em especial se mostraram relevantes: o primeiro, o fato de os Traços de Personalidade do GP não terem impacto direto no Desempenho dos Projetos, porém terem um impacto significativo na dimensão Atitudes do GP, tendo, portanto, um efeito indireto no Desempenho dos Projetos. O segundo ponto são evidências de que a certificação em Gestão de Projetos não afeta diretamente o Desempenho dos Projetos, mas modera as relações entre Habilidades e Conhecimentos e o Desempenho, indícios da forma pela qual as certificações podem influenciar o resultado dos projetos.

Esses resultados ainda abrem campos de pesquisas sobre elementos ligados às características e competências desses profissionais, chamando a atenção dos pesquisadores da área de Administração para investir nos estudos sobre as competências e características pessoais do GP, contribuindo assim para a melhoria dos resultados dos projetos e, consequentemente, nos resultados das organizações. Como trabalho futuro, o modelo de mensuração e o modelo estrutural deste trabalho podem ser replicados em outras pesquisas, para se realizar uma análise confirmatória dos aspectos nele abordados.

Uma limitação deste estudo está ligada ao fato de que a amostra gerada por esta pesquisa teve 
uma maior participação de profissionais de projetos ligados à área de tecnologia da informação e isso pode criar um viés que, de certa foram, pode dificultar a generalização dos achados.

\section{Referências}

Ajzen, I. (2005). Attitudes, personalitty and behavior. Berkshire: Open University Press.

Allport, G. (1961). Pattern and growth in personality. England: Harcourt College Publishers.

Anantatmula, V. (2015). Strategies for enhancing project performance. Journal of Management Engineering, 31(6), 1-8. http://dx.doi.org/10.1061/(ASCE)ME.19435479.0000369 .

Artto, K. A. (2000). What do you manage: processes or personnel's competencies for managing the processes? Project Management Journal, 6(1), 4-9.

Atkinson, R. (1999). Project management: cost, time and quality, two best guesses and a phenomenon, it's time to accept other success criteria. International Journal of Project Management, 17(6), 337-342. http://dx.doi. org/10.1016/S0263-7863(98)00069-6.

Australian Institute of Project Management - AIPM. (1996). National competency standards for project management. Sydney: AIPM.

Bagozzi, R. P., Gurhan-Canli, Z., \& Priester, R. J. (2002). The social psychology of consumer behavior. Philadelphia: Open Universty Press.

Barrick, M. R., \& Mount, M. K. (1991). The Big Five personality dimensions and job performance: a metaanalysis. Personnel Psychology, 44(1), 1-26. http:// dx.doi.org/10.1111/j.1744-6570.1991.tb00688.x.

Barrick, M. R., Mount, M. K., \& Strauss, J. P. (1993). Conscientiousness and performance of sales representatives: Test of the mediating effects of goal setting. The Journal of Applied Psychology, 78(5), 715-722. http://dx.doi. org/10.1037/0021-9010.78.5.715.

Belassi, W., \& Tukel, O. I. (1996). A new framework for determining critical success/failure factors in projects. International Journal of Project Management, 14(3), 141151. http://dx.doi.org/10.1016/0263-7863(95)00064-X.

Bordovskaia, N., \& Kostromina, S. (2013). Personal features and research of potencial students. The European Journal of Social and Behavioural Sciences, 33(12), 1284-1293.

Brandão, H. P. (2012). Mapeamento de competências: métodos, técnicas e aplicações em gestão de pessoas. São Paulo: Atlas.

Brandão, H. P., \& Borges-Andrade, J. E. (2007). Causas e efeitos da expressão competência no trabalho: para entender melhor a noção de competência. Revista de Administração Mackenzie, 8(3), 32-49.

Brasil. Ministério do Trabalho. (2015). Classificação Brasileira de Ocupações: $C B O$. Brasília. Recuperado em 25 de agosto de 2015, de http://www.mtecbo.gov. br/cbosite/pages/home.jsf

Brill, J. M., Bishop, M. J., \& Walker, A. E. (2006). The competencies and characteristics required of an effective project manager: a web-based Delphi study. Association for Educational Communications and Technology, 54(2), 115-140.

Buchaman, D., \& Huczynski, A. (1997). Organizational behaviour: an introductory text (3. ed.). London: Prentice Hall.

Carbone, P., Brandão, H. P., Leite, J. B. D., \& Vilhena, R. M. P. (2009). Gestão por competência e gestão do conhecimento (3. ed.). Rio de Janeiro: Editora FGV.

Cooke-Davies, T. (2002). The "real" success factors on projects. International Journal of Project Management, 20(3), 185-190. http://dx.doi.org/10.1016/S02637863(01)00067-9.

Crawford, L. (2005). Senior management perceptions of project management competence. International Journal of Project Management, 23(1), 7-16. http://dx.doi. org/10.1016/j.ijproman.2004.06.005.

Durand, T. (2006). L'alchimie de la compétence. Revue Française de Gestion., 160, 261-292.

Dutra, J. S. (2001). Gestão do desenvolvimento e da carreira por competência: gestão por competências. um modelo avançado para o gerenciamento de pessoas. São Paulo: Gente.

Ebbesen, J., \& Hope, A. J. (2013). Re-imagining the iron triangle: embedding sustainability into project constraints. Project Management World Journal, 2(3), 1.

El-Sabaa, S. (2001). The skills and career path of an effective project manager. International Journal of Project Management, 19, 183-196.

Fisher, E. (2011). What practitioners consider to be the skills and behaviours of an effective people project manager. International Journal of Project Management, 29(8), 9941002. http://dx.doi.org/10.1016/j.ijproman.2010.09.002.

Fleury, M. T. L. (2002). A gestão de competência e a estratégia organizacional. In M. T. Fleury (Ed.), As pessoas na organização. São Paulo: Gente.

Fotwe, F. T., \& McCaffer, R. (2000). Developing project management competency: perspectives from the construction industry. International Journal of Project Management, 18(2), 111-124. http://dx.doi.org/10.1016/ S0263-7863(98)90075-8.

Freeman, M., \& Beale, P. (1992). Measuring project success. Project Management Journal, 23(1), 8-17.

Gaddis, O. P. (1959). The project manager. Harvard Business Review, 37(3), 89-97.

Goldberg, L. (1990). An alternative "description of personality": the big-five factor structure. Journal of Personality and Social Psychology, 59(6), 1216-1229. PMid:2283588. http://dx.doi.org/10.1037/0022-3514.59.6.1216. 
Gosling, S. D., Rentfrow, P. J., \& Swann, W. B., Jr. (2003). A very brief measure of the Big-Five personality domains. Journal of Research in Personality, 37(6), 504-528. http://dx.doi.org/10.1016/S0092-6566(03)00046-1.

Grygus, I., \& Prusik, K. (2015). Mouding of professional and personal features of a character of future specialists on health. Global Journal of Advanced Research., 2(1), 41-43.

Haggerty, N. (2000). Understanding the link between IT project manager skills and project success research in progress. Illinois: SIGCPR.

Hair, J. F., Jr., Hult, G. T. M., Ringle, C., \& Sarstedt, M. (2015). A primer on partial least squares structural equation modeling (PLS-SEM) (2. ed.). California: Sage.

Hair, J. F., Jr., Ringle, C. M., \& Sarstedt, M. (2011). PLSSEM: indeed, a silver bullet. Journal of Marketing Theory and Practice, 19(2), 39-152. http://dx.doi. org/10.2753/MTP1069-6679190202.

Hair, J. F., Jr., Sarstedt, M., Ringle, C. M., \& Gudergan, S. P. (2014). A primer on partial least squares structural equation modeling (PLS-SEM) (1. ed.). California: Sage.

Hartman, F., \& Skulmoski, G. (1999). Quest for team competence. Project Management Journal, 5(1), 10-15.

Henseler, J., Ringle, C. M., \& Sarstedt, M. (2015). A new criterion for assessing discriminant validity in variance-based structural equation modeling. Journal of the Academy of Marketing Science, 43(1), 115-135. http://dx.doi.org/10.1007/s11747-014-0403-8.

Hurt, M., \& Thomas, J. (2009). Building value through sustainable project management offices. Project Management Journal, 40(1), 55-72. http://dx.doi. org/10.1002/pmj.20095.

Hurtz, G. M., \& Donovan, J. J. (2000). Personality and job performance: the Big Five revisited. The Journal of Applied Psychology, 85(6), 869-879. PMid:11125652. http://dx.doi.org/10.1037/0021-9010.85.6.869.

Ika, L. (2009). Project success as a topic in project management journals. Project Management Journal, 40(4), 6-19. http://dx.doi.org/10.1002/pmj.20137.

Isik, Z., Arditi, D., Dikmen, I., \& Birgonul, M. T. (2009). Impact of corporate strengths / weaknesses on project management competencies. International Journal of Project Management, 27(6), 629-637. http://dx.doi. org/10.1016/j.ijproman.2008.10.002.

Jarvis, C. B., Mackenzie, S. B., \& Podsakoff, P. M. (2003). A critical review of construct indicators and measurement model misspecification in marketing and consumer research. The Journal of Consumer Research, 30(2), 199-218. http://dx.doi.org/10.1086/376806.

John, O. P., \& Srivastava, S. (1999). Big Five Inventory (BFI). In L. A. Pervin \& O. P. John (Eds.), Handbook of personality: theory and research (Vol. 2, pp. 102-138). New York: Guilford Press.
Kerzner, H. (2010). Gestão de projetos: as melhores práticas. São Paulo: Artmed.

Lampel, J. (2001). The core competencies of effective project execution: the challenge of diversity. International Journal of Project Management, 19(8), 471-483.

Le Boterf, G. (2003). Desenvolvendo a competência dos profissionais. Porto Alegre: Bookman-Artmed.

Lechler, T. (1998). When it comes to project management, it's the people that matter: an empirical analysis of project management in Germany. In Hartman, F., Jergeas, G., \& Thomas, J. The nature and role of projects in the next 20 years: research issues and problems. Calgary: University of Calgary.

Lim, C., \& Mohamed, M. (1999). Criteria of project success: an exploratory re-examination. International Journal of Project Management, 17(4), 243-248. http://dx.doi. org/10.1016/S0263-7863(98)00040-4.

Lustri, D., Miuri, I. K., \& Takahachi, S. (2005). Gestão do conhecimento: um modelo conceitual para o desenvolvimento de competências. Inteligência Empresarial, 25, 20-27.

McCrae, R., \& John, O. (1992). An introduction to the five-factor model and its applications. Journal of Personality, 60(2), 175-215. PMid:1635039. http:// dx.doi.org/10.1111/j.1467-6494.1992.tb00970.x.

Milis, K., \& Mercken, R. (2002). Success factors regarding the implementation of ICT investment projects. International Journal of Production Economics, 80(1), 105-117. http:// dx.doi.org/10.1016/S0925-5273(02)00246-3.

Nicholson, N. (2000). Executive instinct: managing the human animal in the information age. New York: Hardcover.

Nunnally, C., \& Bernstein, I. H. (1994). Psychometric theory (3. ed.). San Francisco: McGraw Hill.

O'Brochta, M. (2002). Project success: what are the criteria and whose opinion counts? In Proceedings of the Project Management Institute Annual Seminars and Symposiums. San Antonio: PMI.

Pérez, A., \& Rodríguez del Bosque, I. (2013). Customer personal features as determinants of the formation process of corporate social responsibility perceptions. Psychology and Marketing, 30(10), 903-917. http:// dx.doi.org/10.1002/mar.20654.

Pires, A. K. (2005). Gestão por competências em organizações de governo: mesa redonda de pesquisaação. Brasília: ENAP.

Posner, B. (1987). What it takes to be a good project manager. Project Management Journal, 18(1), 51-54.

Project Management Institute - PMI. (2012). A guide to the project management body of knowledge: Pmbok ${ }^{\circledR}$ guide (5. ed.). Pennsylvania: PMI.

Project Management Institute - PMI. (2013). PMI's industry growth forecast. Pennsylvania: PMI. Recuperado em 20 de abril de 2016, de https:// 
www.pmi.org/ /media/PDF/Business- Solutions/ PMIProjectManagementSkillsGapReport.ashx

Ringle, C. M., Wende, S., \& Becker, J. M. (2015). SmartPLS 3 Boenningstedt: SmartPLS GmbH. Recuperado em 20 de abril de 2016, de http://www.smartpls.com

Sbragia, R., Maximiano, A. C., \& Kruglianskas, I. (1986). O GP: seu papel e habilidades. Revista de Administração, 26(4), 21-24.

Schultz, D. P., \& Schultz, S. E. (2009). História da psicologia moderna. São Paulo: Cengage Learning.

Scott-Young, C., \& Samson, D. (2008). Project success and project team management: evidence from capital projects in the process industries. Journal of Operations Management, 26(6), 749-766. http://dx.doi.org/10.1016/j. jom.2007.10.006.

Soto, C. J., \& John, O. P. (2009). Ten facet scales for the Big Five Inventory: Convergence with NEO PI-R facets, self-peer agreement, and discriminant validity. Journal of Research in Personality, 43(1), 84-90. http://dx.doi. org/10.1016/j.jrp.2008.10.002.

Thal, A. E. Jr., \& Bedingfiel, A. (2010). Successful project managers: an exploratory study into the impact of personality.
Technology Analysis and Strategic Management, 22(2), 243-259. http://dx.doi.org/10.1080/09537320903498587.

Thomas, J., \& Mengel, T. (2008). Preparing project managers to deal with complexity - advanced project management education. International Journal of Project Management, 26(3), 304-315. http://dx.doi.org/10.1016/j. ijproman.2008.01.001.

Turner, J. R., \& Huemann, M. (2001). Project management education in project-oriented societies. Internatinal Project Management Journal, 7(1), 7-13.

Williams, T. (2005). Assessing and moving on from the dominant project management discourse in the light of project overruns. IEEE Transactions on Engineering Management, 52(4), 497-508. http://dx.doi.org/10.1109/ TEM.2005.856572.

Wit, A. (1988). Measurement of project success. International Journal of Project Management, 6(3), 164-170. http:// dx.doi.org/10.1016/0263-7863(88)90043-9.

Zarifian, P. (2003). O modelo da competência: trajetória histórica, desafios atuais e propostas (pp. 192). São Paulo: Senac. 
Apêndice A. Questionário eletrônico.

\begin{tabular}{|c|l|l|}
\hline $\begin{array}{l}\text { Conhecimentos } \\
\text { Tenho conhecimentos em/no }(s) / d o(a)(s)\end{array}$ & \multicolumn{1}{c|}{ Referências } \\
\hline Q1a & Estimativas de projetos & Lampel (2001) \\
\hline Q1b & $\begin{array}{l}\text { Técnicas e ferramentas de gerenciamento de } \\
\text { projetos }\end{array}$ & $\begin{array}{l}\text { Haggerty (2000); Lampel (2001); Fotwe \& McCaffer } \\
(2000) ; \text { Brill et al. (2006) }\end{array}$ \\
\hline Q1c & Medidas de sucesso do projeto & Brill et al. (2006) \\
\hline Q1d & Escrita de propostas & Lampel (2001) \\
\hline Q1e & Ativos de tecnologia & Brill et al. (2006) \\
\hline Q1f & Temas multidisciplinares & Brill et al. (2006); Lampel (2001) \\
\hline Q1g & Da política ou cultura fora da organização & \\
\hline Q1h & Parceiros & \\
\hline
\end{tabular}

\begin{tabular}{|c|l|l|}
\hline $\begin{array}{l}\text { Habilidades } \\
\text { Tenho habilidades em. }\end{array}$ & \multicolumn{1}{c|}{ Referências } \\
\hline Q2a & Aprender & Lampel (2001) \\
\hline Q2b & Criar e inovar & $\begin{array}{l}\text { Fotwe \& McCaffer (2000); Brasil (2015); } \\
\text { Kerzner (2010) }\end{array}$ \\
\hline Q2c & Raciocinar analiticamente & Fotwe \& McCaffer (2000) \\
\hline Q2d & Saber raciocinar logicamente & El-Sabaa (2001) \\
\hline Q2e & Comunicar-me efetivamente & $\begin{array}{l}\text { Fotwe \& McCaffer (2000); Fisher (2011); } \\
\text { Kerzner (2010); El-Sabaa (2001) }\end{array}$ \\
\hline Q2f & Orientar-me por metas & El-Sabaa (2001) \\
\hline Q2g & $\begin{array}{l}\text { Visualizar o relacionamento do projeto com a } \\
\text { indústria e a comunidade }\end{array}$ & El-Sabaa (2001) \\
\hline Q2h & Avaliar situações complexas & Lampel (2001) \\
\hline
\end{tabular}

\begin{tabular}{|c|l|l|}
\hline $\begin{array}{l}\text { Atitudes } \\
\text { Eu ajo }\end{array}$ & \multicolumn{1}{c|}{ Referências } \\
\hline Q3a & De forma organizada & Thomas \& Mengel (2008) \\
\hline Q3b & Compartilhando o crédito pelo sucesso & Brill et al. (2006) \\
\hline Q3c & De forma flexível & El-Sabaa (2001) \\
\hline Q3d & Com persistência & El-Sabaa (2001); Kerzner (2010) \\
\hline Q3e & Com autoestima elevada & El-Sabaa (2001) \\
\hline
\end{tabular}

\begin{tabular}{|c|c|c|}
\hline \multicolumn{2}{|c|}{$\begin{array}{l}\text { Desempenho } \\
\text { Dos meus três últimos projetos, eu obtive sucesso em }\end{array}$} & Referências \\
\hline Q4a & Prazos & \multirow{4}{*}{$\begin{array}{l}\text { Kerzner (2010); Ika (2009); PMI (2012); Ebbesen \& } \\
\text { Hope (2013) }\end{array}$} \\
\hline Q4b & Custos & \\
\hline Q4c & Qualidade & \\
\hline Q4d & Escopo & \\
\hline
\end{tabular}

\begin{tabular}{|c|l|l|}
\hline $\begin{array}{l}\text { Personalidade } \\
\text { Os itens a seguir correspondem a minhas ações e } \\
\text { comportamentos mais frequentes }\end{array}$ & \\
\hline Q5a & Sou aberto ao novo & \\
\hline Q5b & Sou meticuloso e cuidadoso & \multirow{2}{*}{ John \& Srivastava (1999) } \\
\hline Q5c & Sou instável emocionalmente & \\
\hline Q5d & Sou extrovertido & \\
\hline Q5e & Sou amável & \\
\hline
\end{tabular}

\begin{tabular}{|c|l|l|}
\hline \multicolumn{2}{|l|}{ Certificação } & Referências \\
\hline Q6a & $\begin{array}{l}\text { Há quanto tempo você tem ou teve certificação } \\
\text { de gerenciamento de projetos? }\end{array}$ & Hartman \& Skulmoski (1999) \\
\hline
\end{tabular}

